\title{
Dímero $D$ como factor pronóstico de gravedad en pacientes COVID-19
}

\section{D-dimer as a prognostic factor of severity in COVID-19 patients}

Cristel Castillo-Huerta ${ }^{1, a}$, Yiyo Castillo-Velásquez ${ }^{1, a}$, Alicia Chávez-Cerna ${ }^{1, a}$, Anibal Cubas-Iparraguirre, ${ }^{1, a}$, Esteban Vergara-de la Rosa, ${ }^{1, b}$

\begin{abstract}
Señor editor,
El dímero $D(D D)$ es el principal biomarcador específico de la formación y degradación de los coágulos de fibrina, utilizado como herramienta pronóstica del desarrollo de la enfermedad en pacientes COVID-19 ${ }^{(1)}$. Estudios preliminares han afirmado que la mayoría de muertes causadas por el SARS-CoV-2, se asocian frecuentemente a la presencia de alteraciones en la coagulación ${ }^{(2)}$, relacionadas con la progresión y gravedad de la enfermedad $^{(1)}$; incluso, demostraron que los pacientes pueden tener un DD elevado con $46,4 \%$, siendo esta tasa mayor en pacientes con enfermedad grave $(59,6 \%)^{(3)}$. Un estudio adicional, observó un nivel elevado de DD en línea con el progreso de la gravedad del paciente, lo cual reflejaría su papel como factor predictivo en la evolución de la enfermedad ${ }^{(1,4)}$. Lo mencionado anteriormente propone que el uso del valor elevado del DD podría ser un factor pronóstico de gravedad en pacientes COVID-19.
\end{abstract}

En el mismo sentido, un estudio reportó que 138 pacientes mostraron un valor de DD elevado, siendo aproximadamente 2,5 veces superior en aquellos que requirieron cuidados intensivos ${ }^{(5)}$. De igual manera, en un estudio realizado con 221 pacientes, se evidenció que el nivel de DD fue hasta 2,4 veces más predominante en aquellos con mayor compromiso de gravedad $^{(6)}$; así como un análisis superior, que extrajo datos de 1099 pacientes COVID-19 de 552 hospitales de 30 territorios chinos, identificó un aumento significativo de valores de DD en los pacientes con mayor gravedad $^{(7)}$. Tales estudios remarcan que el nivel elevado de DD parece estar relacionado con el pronóstico de gravedad en los pacientes COVID-19.

Sin embargo, existen ciertas limitaciones que pueden influenciar sobre los resultados de los estudios antes mencionados. Las comorbilidades pueden representar

\footnotetext{
1. Facultad de Medicina, Universidad Nacional de Trujillo. Trujillo, Perú.

Estudiante de Pregrado.

b. Docente en medicina. Maestría en investigación clínica.
}

un factor de confusión para la interpretación de los valores del DD, al desconocer las diferencias entre grupos de estudio en cuanto a otras variables clínicas ${ }^{(8)}$. Entre otras limitaciones se encuentran: El sesgo de selección (heterogeneidad elevada entre grupos), un número reducido de pacientes ${ }^{(2)}$, la falta de análisis multivariado en estudios descriptivos de comparación y los diferentes protocolos de medición ${ }^{(9)}$.

Finalmente, podemos concluir que el nivel elevado de DD usado como factor pronóstico de gravedad en pacientes COVID-19, podría permitir identificar el avance de la complicación de la enfermedad y brindar muchos beneficios para establecer soluciones terapéuticas oportunas. Sin embargo, se necesita mayor evidencia de estudios científicos superiores que respalden dicha asociación.

Conflictos de interés: Los autores niegan conflictos de interés.

Financiamiento: Autofinanciado.

\section{REFERENCIAS BIBLIOGRÁFICAS}

1. Kariyanna P, Aurora L, Jayarangaiah A, Yadav V, Hossain NA, Akter N, et al. Utility of D-dimer as a prognostic factor in SARS CoV2 infection: A review. Am J Med Case Rep [Internet]. 2020;8(10):337-40. A va i l a bler from : https://www.ncbi.nlm.nih.gov/pmc/articles/PM C7447555/

2. Moreno G, Carbonell R, Bodí M, Rodríguez A. Revisión sistemática sobre la utilidad pronóstica del dímero-D, coagulación intravascular diseminada y tratamiento anticoagulante en pacientes graves con COVID-19. Med Intensiva [Internet]. 2020;S0210-5691(20):30187-X. A v a i l a ble f rom : https://www.ncbi.nlm.nih.gov/pmc/articles/PM C7298463/

3. Giannis D, Ziogas IA, Gianni P. Coagulation disorders in coronavirus infected patients: COVID- 
19, SARS-CoV-1, MERS-CoV and lessons from the past. J Clin Virol [Internet]. 2020;127:104362. A $v$ a i l a b l e f r o m : https://www.sciencedirect.com/science/article /abs/pii/S1386653220301049

4. Han H, Yang L, Liu R, Liu F, Wu KL, Li J, et al. Prominent changes in blood coagulation of patients with SARS-CoV-2 infection. Clin Chem Lab Med [Internet]. 2020;58(7):1116-20. Available https://pubmed.ncbi.nlm.nih.gov/32172226/\#: :text=The values of $\mathrm{D}$-dimer, in patients with milder forms.

5. Wang D, Hu B, Hu C, Zhu F, Liu X, Zhang J, et al. Clinical Characteristics of 138 Hospitalized Patients With 2019 Novel Coronavirus-Infected Pneumonia in Wuhan, China. JAMA [Internet]. 2020;323(11):1061-9. Available from: https://www.ncbi.nlm.nih.gov/pmc/articles/PM C7042881/

6. Zhang G, Hu C, Luo L, Fang F, Chen Y, Li J, et al. Clinical features and short-term outcomes of 221 patients with COVID-19 in Wuhan, China. J Clin Virol [Internet]. 2020;127:104364. Available from: https://doi.org/10.1016/j.jcv.2020.104364

7. Guan W, Ni Z, Hu Y, Liang W, Ou C, He J, et al. Clinical characteristics of coronavirus disease 2019 in China. N Engl J Med [Internet]. 2020;382(18):1708-20. Available from: https://www.thelancet.com/journals/lancet/art
icle/PIIS0140-6736(20)30183-5/fulltext

8. Cho ES, Mcclelland PH, Cheng O, Kim Y, Hu J, Zenilman ME, et al. Utility of d-dimer for diagnosis of deep vein thrombosis in coronavirus disease-19 infection. J Vasc Surg Venous Lymphat Disord [Internet]. 2020;S2213-333X(20)30420-0. A vailable from : https://www.ncbi.nlm.nih.gov/pmc/articles/PM C7390766/\#: : :text=Key Findings\%3A In 158 coronavirus, diagnosis of deep vein thrombosis.

9. Vidali S, Morosetti D, Cossu E, Luisi M, Pancani S, Semeraro V, et al. D-dimer as an indicator of prognosis in SARS-CoV-2 infection: a systematic review. ERJ Open Res [Internet]. 2020;6(2):00260-2020. Available from: https://www.ncbi.nlm.nih.gov/pmc/articles/PM C7357271/.

\section{Correspondencia}

Cristel Castillo-Huerta

Dirección: Facultad de Medicina, Universidad Nacional de Trujillo. Trujillo-Perú. Ramón Castilla Mz. B Lt. 45 Urb. 20 de Abril, Trujillo, Perú.

Teléfono: 51-940175432

Correo: ccastilloh@unitru.edu.pe

\section{Revisión de pares}

Recibido: $18 / 10 / 2020$

Aceptado: 15/12/2020 